\title{
Critical behavior of the Widom-Rowlinson mixture: coexistence diameter and order parameter
}

\author{
R. L. C. Vink \\ Institut für Theoretische Physik II, Heinrich Heine Universität Düsseldorf, \\ Universitätsstraße 1, 40225 Düsseldorf, Germany
}

(Dated: August 6, 2018)

\begin{abstract}
The critical behavior of the Widom-Rowlinson mixture [J. Chem. Phys. 52, 1670 (1970)] is studied in $d=3$ dimensions by means of grand canonical Monte Carlo simulations. The finite size scaling approach of Kim, Fisher, and Luijten [Phys. Rev. Lett. 91, 065701 (2003)] is used to extract the order parameter and the coexistence diameter. It is demonstrated that the critical behavior of the diameter is dominated by a singular term proportional to $t^{1-\alpha}$, with $t$ the relative distance from the critical point, and $\alpha$ the critical exponent of the specific heat. No sign of a term proportional to $t^{2 \beta}$ could be detected, with $\beta$ the critical exponent of the order parameter, indicating that pressuremixing in this model is small. The critical density is measured to be $\rho \sigma^{3}=0.7486 \pm 0.0002$, with $\sigma$ the particle diameter. The critical exponents $\alpha$ and $\beta$, as well as the correlation length exponent $\nu$, are also measured and shown to comply with $d=3$ Ising criticality.

PACS numbers: 02.70.-c, 05.70.Jk, 64.70.Fx, 64.60.Fr
\end{abstract}

\section{INTRODUCTION}

The Widom-Rowlinson (WR) mixture $\frac{1}{\underline{\underline{m}}}$ is a simple model of a fluid exhibiting phase separation. The model consists of A and B particles that interact via simple pair potentials: the AA and BB pair interaction is ideal, while $\mathrm{AB}$ pairs interact via a hard-core potential of diameter $\sigma$. Upon increasing density, the WR mixture phase separates into an A-rich and B-rich phase. In $d=3$ dimensions, computer simulations agree that the corresponding universality class is that of the three-dimensional (3D) Ising mode ${ }^{2.3 .4 .5}$. There is, however, some variation in the reported estimates of the critical density.

In order to probe fluid criticality using computer simulation, high quality data are required. The latter are typically generated using Monte Carlo (MC) methods, and considerable effort has been devoted to develop efficient MC schemes. In Ref. 3, for example, a MC cluster move is described for the WR mixture that is (nearly) free of critical slowing down. However, as pointed out in Ref. 5, this type of move cannot be used to obtain the coexistence curve, which may be problematic if one is interested in measuring, say, the critical exponent $\beta$ of the order parameter. In Ref. 5, therefore, a different cluster move is formulated, based on Ref. 6 , which not only gives access to the coexistence curve, but is also rejection free. Recently, the latter approach was generalized to continuous potentials ${ }^{7}$.

However, in addition to efficient MC sampling, of at least equal importance (if not more) is the finite size scaling (FSS) algorithm used to extrapolate the simulation data to the thermodynamic limit. FSS is essential because the correlation length diverges at the critical point, and thus the true thermodynamic limit is never captured in a finite simulation box, no matter how efficiently it is simulated. For fluids, recently proposed unbiased FSS algorithms formulated in the grand canonical ensemble seem particularly powerfu1 ${ }^{8.9 .10 .11}$. The latter algorithms are unbiased in the sense that no prior knowledge of the universality class is required: the critical point of the transition, as well as some of the critical exponents, are an output. These unbiased algorithms were used, for example, to resolve the universality class of the hard-core square-well (HCSW) fluid and the restricted primitive electrolyte, both of which were shown to exhibit 3D Ising critical behavior ${ }^{9.12}$.

Unfortunately, it is not obvious how the MC cluster moves for the WR mixture generalize to the grand canonical ensemble. Grand canonical cluster moves for mixtures seem less common, but some have been presented in the literature $\frac{13.14}{2}$. Of these, the MC move of Ref. 14, a generalization of its canonical variant 15 , is readily applicable to the WR mixture. By using the MC move of Ref. 14, a FSS analysis of the WR mixture using the above mentioned unbiased algorithms thus becomes possible. This, consequently, is the aim of the present work. Of particular interest is the coexistence diameter, whose critical behavior is governed by a very weak singularity that is challenging to extract from simulation data. Note that, at the time of writing, the approach of Ref. 9 seems to be the only FSS algorithm available to extract the coexistence diameter correctly from simulation data. A correct description of the latter is required in order to reliably estimate the critical density ${ }^{16}$. Since the coexistence diameter of the WR model has not received much attention in previous simulations, the present grand canonical approach is certainly warranted.

\section{SIMULATION METHOD}

In the grand canonical ensemble, the volume $V$ and the fugacity $z_{\mathrm{A}}\left(z_{\mathrm{B}}\right)$ of species $\mathrm{A}(\mathrm{B})$ are fixed, while the particle numbers $N_{\mathrm{A}}$ and $N_{\mathrm{B}}$ fluctuate. The thermal wavelength is set to unity such that the fugacity $z_{\alpha}$ directly reflects the number density $N_{\alpha} / V$ a pure phase of 
$\alpha$ particles would have (recall that such a phase is simply an ideal gas). In what follows, all remaining length scales are expressed in terms of the hard-core diameter $\sigma$. The crucial quantity is the finite-size grand canonical distribution $P_{L}\left(N_{\mathrm{A}}, N_{\mathrm{B}} \mid z_{\mathrm{A}}, z_{\mathrm{B}}\right)$, defined as the probability of observing a system containing $N_{\mathrm{A}}$ particles of species A and $N_{\mathrm{B}}$ particles of species $\mathrm{B}$, at fugacities $z_{\mathrm{A}}$ and $z_{\mathrm{B}}$, with $L$ the lateral dimension of the cubic simulation box (the use of periodic boundary conditions is assumed).

The distribution is obtained numerically in a grand canonical MC simulation via the insertion and removal of particles. To simulate efficiently, the cluster move of Ref. 14 is used; $P_{L}\left(N_{\mathrm{A}}, N_{\mathrm{B}} \mid z_{\mathrm{A}}, z_{\mathrm{B}}\right)$ is then obtained simply by maintaining a histogram. To overcome the free energy barrier separating the phases, a biased sampling scheme is also implemented 17 . Here, the simulation is divided into distinct intervals (called windows) each spanning a single A particle, while $N_{\mathrm{B}}$ is allowed to fluctuate freely inside each window (the choice for A or B is arbitrary). The windows are then sampled separately and successively. CPU time is divided such that the number of "sweeps" per window is the same for all windows. In this work, we say that a sweep has passed when a given population of particles has completely been replaced or updated by new ones. This is in contrast to the more common approach of keeping the number of attempted MC moves per window fixed. The latter approach, however, is less appropriate for grand canonical simulations since the acceptance rate is typically density dependent. Per window, approximately 1800 sweeps are generated. To obtain a single distribution, an investment of around 7 CPU hours for a small system $(L=8)$, and 270 hours for a large system $(L=13)$ is required. In order to perform the subsequent FSS analysis, $P_{L}\left(N_{\mathrm{A}}, N_{\mathrm{B}} \mid z_{\mathrm{A}}, z_{\mathrm{B}}\right)$ is measured for system sizes $L=8-13$ at fugacities ranging from close to the critical point to well into the coexistence region. Estimates of properties at intermediate fugacities are obtained using the multiple histogram method ${ }^{18}$.

\section{RESULTS}

As mentioned before, the WR mixture exhibits phase separation into an A-rich and B-rich phase. Note that the B-rich phase may equally well be regarded as being poor in A species. In this sense, then, phase separation is analogous to liquid-vapor coexistence: the A-rich phase being the liquid, the A-poor phase being the vapor, and the fugacity of the $\mathrm{B}$ particles being inverse temperature (again, the choice for A or B is arbitrary). A natural definition of the order parameter is therefore $\Delta \equiv\left(\rho_{\mathrm{L}}-\rho_{\mathrm{V}}\right) / 2$, with $\rho_{\mathrm{L}}$ the number density of A particles in the A-rich phase, and $\rho_{\mathrm{V}}$ the number density of A particles in the A-poor phase. Close to the critical point, the order parameter is expected to scale as $\Delta \propto t^{\beta}$, with $t=z_{\mathrm{B}} / z_{\mathrm{B}, \mathrm{cr}}-1$ the distance from the critical point, and $z_{\mathrm{B}, \mathrm{cr}}$ the critical "inverse temperature". Similarly, the coexistence diameter can be written as $D \equiv\left(\rho_{\mathrm{L}}+\rho_{\mathrm{V}}\right) / 2$.
The critical behavior of the latter is given by 19

$$
D=\rho_{\mathrm{A}, \mathrm{cr}}\left(1+A_{2 \beta} t^{2 \beta}+A_{1-\alpha} t^{1-\alpha}+A_{1} t\right),
$$

with $\rho_{\mathrm{A}, \mathrm{cr}}$ the number density of A particles at the critical point, and non-universal amplitudes $A_{i}$. For the 3D Ising universality class, appropriate exponent values are $\beta \approx$ 0.326 and $\alpha \approx 0.109^{20}$.

\section{A. Order parameter}

To extract the order parameter, the FSS algorithm of Ref. 9 is used (for a more detailed description of the algorithm, Ref. 10 is also highly recommended). The algorithm requires as input the grand canonical distribution $P_{L}\left(N_{\mathrm{A}}, N_{\mathrm{B}} \mid z_{\mathrm{A}}, z_{\mathrm{B}}\right)$ for at least three system sizes $L$. Here, five system sizes $L=9,10,11,12,13$ are in fact used. Starting with $z_{\mathrm{B}}$ significantly above its critical value, the cumulant ratio $\left\langle m^{2}\right\rangle^{2} /\left\langle m^{4}\right\rangle$ is plotted as function of the average number density $\left\langle N_{\mathrm{A}}\right\rangle / V$, with $m=N_{\mathrm{A}}-\left\langle N_{\mathrm{A}}\right\rangle$ (note that this plot is parameterized by the fugacity of the other species $z_{\mathrm{A}}$ ). The resulting curve will reveal two minima, located at $\rho^{-}$and $\rho^{+}$, with respective values $Q^{-}$and $Q^{+}$at the minima. Defining the quantities $Q_{\min }=\left(Q^{+}+Q^{-}\right) / 2, x=Q_{\min } \ln \left(4 / e Q_{\min }\right)$, and $y=\left(\rho^{+}-\rho^{-}\right) /(2 \Delta)$, the points $(x, y)$ from the different system sizes should, in the limit far away from the critical point, collapse onto the line $y=1+x / 2$. Recall that $\Delta$ is the order parameter in the thermodynamic limit at the considered fugacity $z_{\mathrm{B}}$, precisely the quantity of interest, which may thus be obtained by fitting until the best collapse onto $1+x / 2$ occurs. In the next step, $z_{\mathrm{B}}$ is chosen closer to the critical point, the points $(x, y)$ are calculated as before, but this time $\Delta$ is chosen such that the new data set joins smoothly with the previous one, yielding an estimate of the order parameter at the new fugacity. This procedure is repeated all the way to the critical point, where $\Delta$ vanishes, leading to an estimate of the critical fugacity $z_{\mathrm{B}, \mathrm{cr}}$. Moreover, the procedure also yields $y$ as function of $x$. The latter scaling function is universal within a universality class, and for the HCSW fluid can be found in Ref. 9. Since the WR mixture belongs to the same universality class, a similar curve should be found. The latter is verified in Fig. 11 which shows $y$ as function of $x$ obtained in this work, compared to the result of Ref. 9 . The agreement is very reasonable. From the vanishing of the scaling function, at $x_{c}=0.280$, an unbiased estimate of the critical fugacity $z_{\mathrm{B}, \mathrm{cr}}=0.93791 \pm 0.00004$ is obtained. Note that $x_{c}$ is universal within a universality class. The estimate reported here compares favorably to $x_{c}=0.286$ obtained for the HCSW fluid ${ }^{9}$, and $x_{c}=0.296$ obtained for the 3D Ising model21, providing additional confirmation that these systems belong to the same universality class. Shown in Fig. 2] on double logarithmic scales, is the order parameter $\Delta$ of the WR mixture as function of the distance from the critical point $t$, where the above quoted estimate of $z_{\mathrm{B}, \mathrm{cr}}$ was used. The resolution of the present 
data is such that $\Delta$ can be resolved down to $t \approx 5 \times 10^{-5}$. By fitting the lowest few points in Fig. 2 to $\Delta \propto t^{\beta}$, the critical exponent is measured to be $\beta \approx 0.322 \pm 0.008$, which is certainly compatible with the accepted 3D Ising value.

\section{B. Coexistence diameter}

To extract the coexistence diameter, the FSS algorithm of Ref. 11 is used. The algorithm is similar in spirit to the previous one, in the sense that it generates a scaling function $y=f(x)$, starting with data obtained well away from the critical point, and then recursively working its way down toward criticality. In Fig. 3 the scaling function of the diameter for the WR mixture thus obtained is shown, where, as before, five system sizes $L=9-13$ were used. For $x \rightarrow 0$, this function is expected to approach $y=x / 2$, which indeed it does. In contrast to the order parameter, however, the scaling function of the coexistence diameter is not universa111. Therefore, a direct comparison to scaling functions of other systems cannot, in general, be carried out. Nevertheless, for systems with negligible pressure mixing, such as the HCSW fluid and presumably also the WR mixture, the scaling function is expected to be well described by the approximant 11

$$
\begin{aligned}
e_{l}(x)= & C_{l}\left[1-(1-\bar{x})^{1-\alpha}\right. \\
& \left.\times \frac{1+s_{1} \bar{x}+s_{2} \bar{x}^{2}+s_{3} \bar{x}^{3}}{1+t_{1} \bar{x}+t_{2} \bar{x}^{2}+t_{3} \bar{x}^{3}}\right],
\end{aligned}
$$

with $\bar{x}=x / x_{c}, t_{1}=s_{1}-1+\alpha+x_{c} / 2 C_{l}$, and critical exponent $\alpha \approx 0.109$. A fit to the WR data of Fig. 3 shows that this is indeed the case, with explicit parameter values $C_{l}=0.429, x_{c}=0.175, s_{1}=4.50, s_{2}=-5.72$, $s_{3}=0.12, t_{1}=3.81, t_{2}=-9.08$ and $t_{3}=4.25$. These values are remarkably consistent with estimates quoted in Ref. 11 for the HCSW fluid. Note that $e_{l}(x)$ becomes singular close to $x_{c}$, implied by the $(1-\bar{x})^{1-\alpha}$ factor in Eq.(2). The latter would yield a vertical tangent in the plot, at the arrow in Fig. 3. The present simulation data, however, seem not to extend close enough to the critical point to reach this regime.

The critical behavior of the coexistence diameter $D$ is shown in Fig. 4. where $z_{\mathrm{B}, \mathrm{cr}}=0.93791$ obtained in the previous paragraph was used. In order to facilitate the comparison to other work, $2 \times D$ is actually plotted. Symmetry considerations ensure equal numbers of $\mathrm{A}$ and $\mathrm{B}$ particles at criticality, such that the overall critical number density equals $\rho_{\mathrm{cr}}=2 \rho_{\mathrm{A}, \mathrm{cr}}$, which is the quantity usually quoted in the literature. A fit to the asymptotic expansion of Eq.(1) yields $\rho_{\mathrm{cr}}=0.7486 \pm 0.0002$, where the error reflects the variation stemming from the range over which the fit is performed (repeating the entire analysis leaving out the smallest system size yields a similar result). The corresponding amplitudes read as $A_{2 \beta} \approx 0$, $A_{1-\alpha}=2.76 \pm 0.07$ and $A_{1} \approx-1.27 \pm 0.09$, implying that the singular behavior is dominated by $t^{1-\alpha}$. This, in combination with the observation that the scaling function is well described by $e_{l}(x)$, confirms that pressure mixing in the WR mixture is small. The curvature of the diameter close to the critical point thus reflects the $t^{1-\alpha}$ singularity. Since $1-\alpha$ is close to unity, and the magnitudes of $A_{1-\alpha}$ and $A_{1}$ are similar, the curvature is hard to see in Fig. 4. The singular behavior of the diameter can be visualized nevertheless by plotting the "inverse temperature" derivative $\kappa=2 d D / d t$ instead. In case of singular behavior, $\kappa$ is expected to diverge when $t \rightarrow 0$, see Eq.(1). Though not very precise, this procedure even allows for an unbiased measurement of the exponent $\alpha$. The result is summarized in the inset of Fig. 4 which shows $\kappa$ as function of $t$, where again $z_{\mathrm{B}, \mathrm{cr}}=0.93791$ in $t$ was used. The divergence is clearly visible. By fitting the data to the form $\kappa=a_{1} t^{-\alpha}+a_{2}$, with fit parameters $a_{i}$ and $\alpha$, the specific heat exponent is measured to be $\alpha=0.11 \pm 0.02$, which is surprisingly close to the $3 \mathrm{D}$ Ising value.

\section{Cumulant intersections}

For the sake of completeness, and also to check the consistency of the results obtained so far, the critical fugacity $z_{\mathrm{B}, \mathrm{cr}}$ is measured again, but this time around using the cumulant intersection approach ${ }^{22}$. As was shown by Binder ${ }^{22}$, the (for example) first order cumulant $U_{1}=\left\langle m^{2}\right\rangle /\langle|m|\rangle^{2}$ becomes system-size independent at the critical point. Plots of $U_{1}$ as function of $z_{\mathrm{B}}$ for different system sizes are thus expected to show a common intersection point, leading to an unbiased estimate of the critical fugacity. Moreover, the cumulant value $Q_{c}$ at the intersection point is universal, dependent only on the universality class. Shown in Fig. 5] is the result of this procedure, on a rather fine scale. The resulting estimate reads as $z_{\mathrm{B}, \mathrm{cr}}=0.9379 \pm 0.0004$, where the error reflects the scatter in the intersection points. The latter is fully consistent with the previous, more precise value, $z_{\mathrm{B}, \mathrm{cr}}=0.93791 \pm 0.00004$ (arrow in Fig. 5). For the critical value of the cumulant $Q_{c} \approx 1.223$ is obtained. This value compares quite favorably to the estimate $Q_{c}=1.2391 \pm 0.0014$ obtained in large-scale simulations of the $3 \mathrm{D}$ Ising lattice mode ${ }^{23}$, deviating from it by less than $2 \%$. The inset of Fig. 5 shows the slope of the cumulant $Y_{1}=\left|\mathrm{d} U_{1} / \mathrm{d} z_{\mathrm{B}}\right|$ at the critical fugacity, as function of the system size $L$. It is expected that $Y_{1} \propto L^{1 / \nu}$ with $\nu$ the critical exponent of the correlation length. Although there is some scatter in the intersection points, the cumulant slopes seem rather constant over the range of Fig. [5] and so it is expected that $\nu$ can be obtained quite reliably nevertheless. Indeed, by performing a fit to the data in the inset of Fig. 5 the exponent is measured to be $\nu \approx 0.630 \pm 0.005$, in excellent agreement with the accepted $3 \mathrm{D}$ Ising value $\nu_{\mathrm{Is}} \approx 0.630^{20}$. 


\section{DISCUSSION AND SUMMARY}

In this work, the critical behavior of the WR mixture was investigated using grand canonical MC simulations and unbiased FSS algorithms. As expected, the universality class of the transition is that of the 3D Ising model. This was demonstrated by direct measurements of the exponents $\alpha, \beta$ and $\nu$. Substantial indirect evidence has also been provided, by comparing the scaling function of the order parameter and the coexistence diameter to those of the HCSW fluid, as well as via the universality of $Q_{c}$ at the cumulant intersection point.

The critical density obtained in this work $0.7486 \pm$ 0.0002 can be compared to other simulations. Shew and Yethiraj report $0.762 \pm 0.016^{2}$ using semigrand simulations 24.25 , while Johnson et al. (JEA) obtained $0.748 \pm 0.002^{3}$. More recent estimates are due to Góźdź, $0.759 \pm 0.019^{4}$ using the Bruce-Wilding field mixing technique ${ }^{26}$, and Buhot $0.7470 \pm 0.0008^{5}$. Of the above, JEA and Buhot are very close to the value reported here, see Fig. 4 The estimate of Góźdź, however, is higher. A possible explanation is the adapted FSS algorithm in the latter, which seems to overestimate the critical density in some cases ${ }^{16}$. JEA also report an estimate of the critical fugacity $z_{\mathrm{B}, \mathrm{cr}} \approx 0.9403$ for the largest system size considered by them ${ }^{3}$, but without a systematic FSS analysis of this quantity. This overestimates the present value significantly. Interestingly, these authors observe an increase of $z_{\mathrm{B} \text {,cr }}$ with system size, in disagreement with the present work.

Buhot, by using rejection-free cluster MC moves, is able to simulate impressively large systems ${ }^{5}$, up to $L=$ 100 , which exceeds the typical system size of the present investigation by about one order of magnitude. The improved accuracy of the critical density obtained in this work may therefore seem surprising. It should be emphasized, however, that critical phenomena are most conveniently studied in terms of a field variable, such as temperature or, in the case of the WR mixture, the fugacity. The ensemble used by Buhot, as well as the semigrand ensemble, do not have access to the fugacity. Instead, in these ensembles, the critical point is approached by varying the overall density $\rho=\left(N_{\mathrm{A}}+N_{\mathrm{B}}\right) / V$. This somewhat restricts the investigation of critical phenomena because for every considered $\rho$, an explicit simulation needs to be carried out. In grand canonical simulations, on the other hand, one has access to the particle fugacities. This facilitates the extrapolation of simulation data obtained at one set of fugacities to different values via histogram reweighting 18 . Clearly, the investigation of subtle effects, such as the critical behavior of the coexistence diameter, is not really feasible without such extrapolation methods. Note that the behavior depicted in Fig. 4 is not simply an "artifact" of the grand canonical ensemble. In the semigrand ensemble, for example, the singular behavior of the diameter leads to a renormalization of the critical exponent $\beta^{27}$. Assuming negligible $A_{2 \beta}$ in Eq.(1), one obtains $\rho / \rho_{\text {cr }}-1 \propto t^{1-\alpha}$ close to the critical point. Combining this with the critical power law of the order parameter $\Delta \propto t^{\beta}$ and eliminating $t$, yields $\Delta \propto\left(\rho / \rho_{\mathrm{cr}}-1\right)^{\beta^{\star}}$, with renormalized exponent $\beta^{\star}=\beta /(1-\alpha)$. The latter renormalized exponent has been confirmed experimentally ${ }^{28}$, and should, in principle, also show up in the WR mixture when, as mentioned above, the critical point is approached by varying $\rho$.

Needless to say, the WR mixture has also been studied by theoretical means, using for example density functional theory ${ }^{29}$, and integral equations ${ }^{2.30}$. These investigations, however, all pertain to the mean-field level. As such, quantitative agreement with computer simulations close to criticality is not to be expected, and a comparison is consequently not carried out.

\section{Acknowledgments}

This work was supported by the Deutsche Forschungsgemeinschaft under the SFB-TR6 (project section D3). I also thank $\mathrm{K}$. Binder for an initial reading of the manuscript.
1 B. Widom and J. S. Rowlinson, J. Chem. Phys. 52, 1670 (1970).

2 C.-Y. Shew and A. Yethiraj, J. Chem. Phys. 104, 7665 (1996).

3 G. Johnson, H. Gould, J. Machta, and L. K. Chayes, Phys. Rev. Lett. 79, 2612 (1997).

${ }^{4}$ W. T. Góźdź, J. Chem. Phys. 122, 074505 (2005).

5 A. Buhot, J. Chem. Phys. 122, 024105 (2005).

6 A. Buhot and W. Krauth, Phys. Rev. Lett. 80, 3787 (1998).

7 J. Liu and E. Luijten, Phys. Rev. Lett. 92, 035504 (2004).

8 G. Orkoulas, M. E. Fisher, and A. Z. Panagiotopoulos, Phys. Rev. E 63, 051507 (2001).

${ }^{9}$ Y. C. Kim, M. E. Fisher, and E. Luijten, Phys. Rev. Lett. 91, 065701 (2003).
10 Y. C. Kim and M. E. Fisher, Comput. Phys. Commun. 169, 295 (2005).

11 Y. C. Kim, Phys. Rev. E 71, 051501 (2005).

12 E. Luijten, M. E. Fisher, and A. Z. Panagiotopoulos, Phys. Rev. Lett. 88, 185701 (2002).

13 G. Orkoulas and A. Z. Panagiotopoulos, J. Chem. Phys. 101, 1452 (1994).

14 R. L. C. Vink and J. Horbach, J. Chem. Phys. 121, 3253 (2004).

15 T. Biben, P. Bladon, and D. Frenkel, J. Phys.: Condens. Matter 8, 10799 (1996).

16 Y. C. Kim and M. E. Fisher, J. Phys. Chem. B 108, 6750 (2004).

17 P. Virnau and M. Müller, J. Chem. Phys. 120, 10925 (2004). 
18 A. M. Ferrenberg and R. H. Swendsen, Phys. Rev. Lett. 63, 1195 (1989).

19 Y. C. Kim, M. E. Fisher, and G. Orkoulas, Phys. Rev. E 67, 061506 (2003).

${ }^{20}$ M. E. Fisher and S.-Y. Zinn, J. Phys. A: Math. Gen. 31, L629 (1998).

21 M. M. Tsypin and H. W. J. Blöte, Phys. Rev. E 62, 73 (2000).

22 K. Binder, Z. Phys. B: Condens. Matter 43, 119 (1981).

${ }^{23}$ E. Luijten, Phys. Rev. E 59, 4997 (1999).

24 H.-P. Deutsch and K. Binder, Macromolecules 25, 6214 (1992).

25 E. de Miguel, E. Martín de Río, and M. M. Telo da Gama, J. Chem. Phys. 103, 6188 (1995).

26 A. D. Bruce and N. B. Wilding, Phys. Rev. Lett. 68, 193 (1992).

27 M. E. Fisher, Phys. Rev. 176, 257 (1968).

28 B. H. Chen, B. Payandeh, and M. Robert, Phys. Rev. E 62, 2369 (2000).

29 M. Schmidt, Phys. Rev. E 63, 010101 (2001).

30 A. Yethiraj and G. Stell, J. Stat. Phys. 100, 39 (2000).

\section{FIGURE CAPTIONS}

FIG. 1: Scaling function of the order parameter. Following the convention of Ref. 9, the scaling function is raised to a negative exponent, with $\phi=1 / \beta$ and $\beta=0.326$. The solid curve is the result obtained in this work for the WR mixture; the dashed curve is the HCSW result of Ref. 9. Also shown is the exact small $x$ limiting form $y=1+x / 2$.

FIG. 2: Order parameter of the WR mixture as function of the distance from the critical point. The dashed line has a slope $\beta=0.326$, corresponding to the 3D Ising exponent.

FIG. 3: Scaling function of the coexistence diameter for the WR mixture. Open circles show simulation results obtained using the FSS algorithm of Ref. 11. The dashed curve is a fit to the simulation data using the approximant of Eq.(2). Also shown is the exact small $x$ limiting form $y=x / 2$.

FIG. 4: Coexistence diameter of the WR mixture as function of the distance from the critical point. Open circles are simulation results obtained in this work using the FSS algorithm of Ref. 11. The dashed curve is a fit to Eq.(11). The black dot marks the critical density obtained from the fit, where the vertical line indicates the uncertainty. The arrows mark estimates of $\rho_{\text {cr }}$ reported in Ref. 3 (JEA) and Ref. 5 (Buhot), where the vertical lines again indicate the uncertainty. The inset shows $\kappa$ as function of $t$. Open circles are simulation results; the dashed curve, which essentially overlaps the simulation data, is a three-parameter fit of the form $\kappa=a_{1} t^{-\alpha}+a_{2}$, with fit parameters $a_{i}$ and $\alpha$.

FIG. 5: Cumulant analysis of the WR mixture. Shown is the first order cumulant $U_{1}$ as function of the fugacity $z_{\mathrm{B}}$ for various system sizes $L$ as indicated. The inset shows the slope of the cumulant $Y_{1}$ at the critical point as function of $L$. All data were obtained along the symmetry locus $z_{\mathrm{A}}=z_{\mathrm{B}}$. 


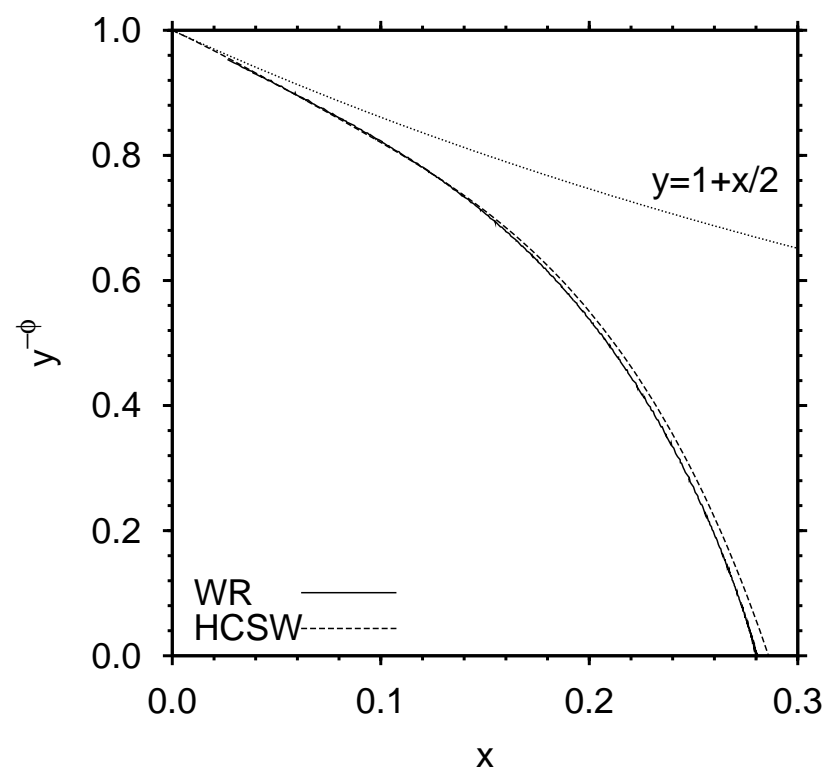

FIG. 1:

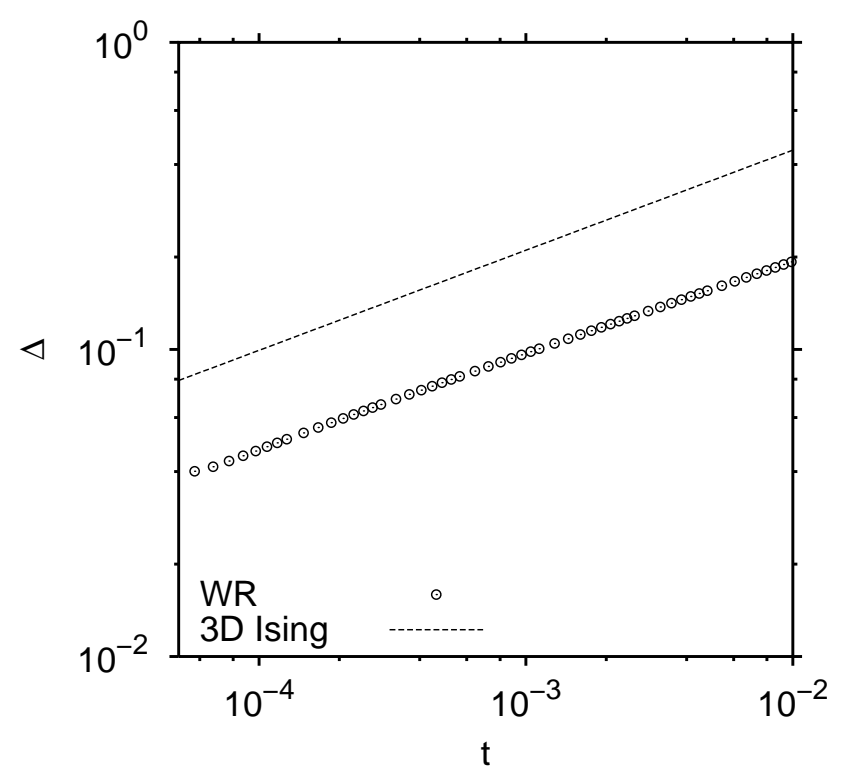

FIG. 2: 


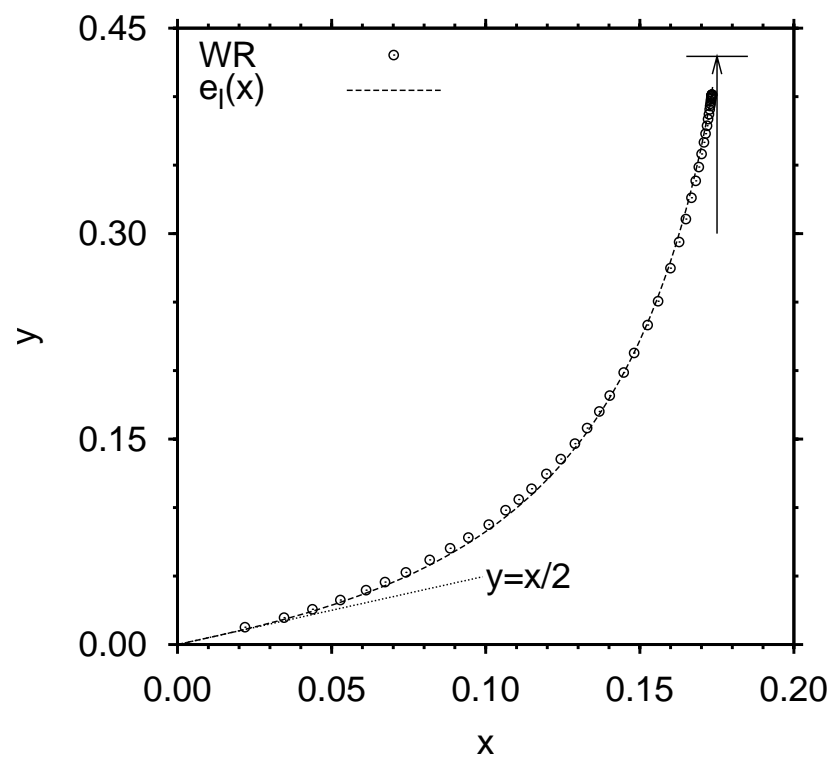

FIG. 3:

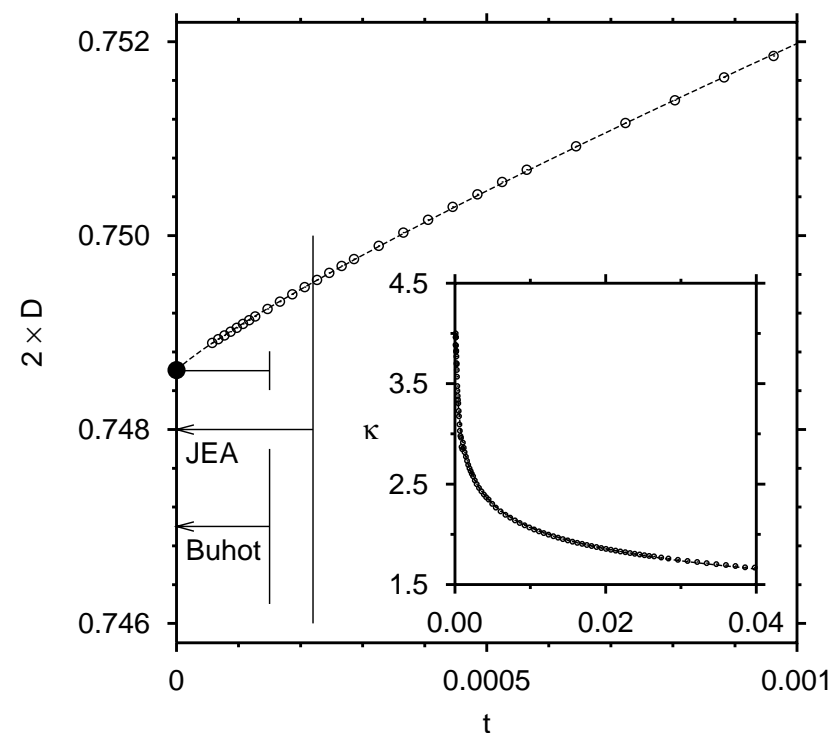

FIG. 4: 


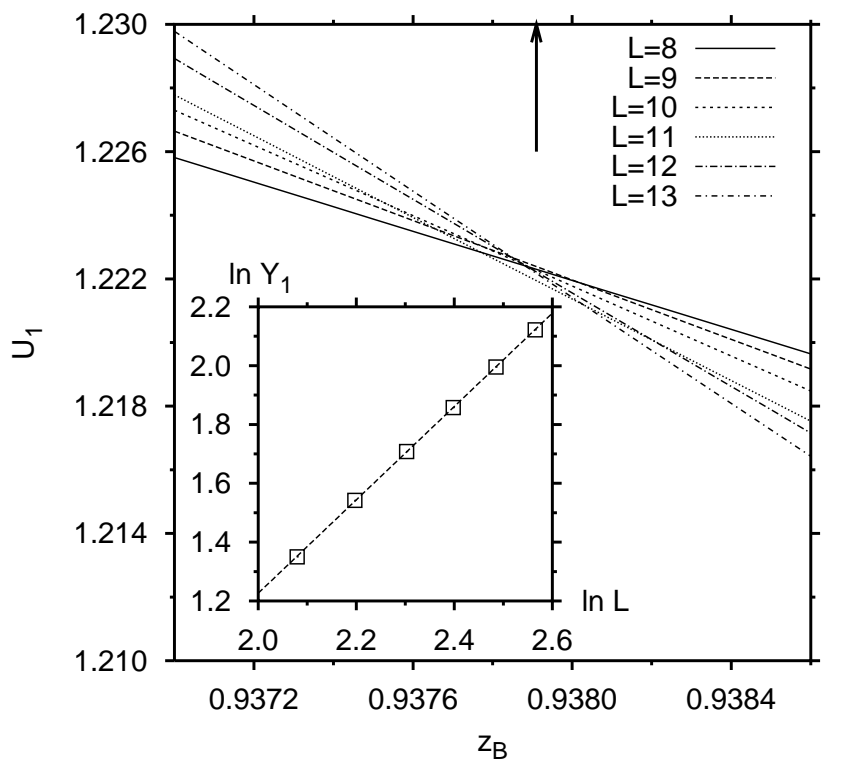

FIG. 5: 